\title{
Assembly and interaction of polyaniline chains: impact on electro- and physical-chemical behavior
}

Elena Tomšík, ${ }^{* a}$ Olena Kohut, ${ }^{a}$ Iryna Ivanko, ${ }^{a}$ Michal Pekárek, ${ }^{a}$ Igor Bieloshapka, ${ }^{b}$ and Panagiotis Dallas

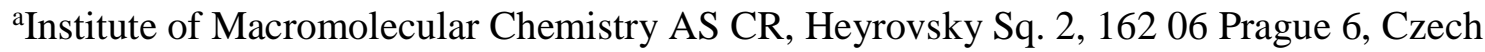
Republic

bInstitute of Physics AS CR, Cukrovarnická 10/112, 16200 Prague 6, Czech Republic

'Department of Materials, Parks Road, University of Oxford, OX1 3PH, United Kingdom

\section{AUTHOR INFORMATION}

\section{Corresponding Author}

* Elena Tomšík, E-mail: tomsik@imc.cas.cz

KEYWORDS. polyaniline; nanofibrils; pseudocapacitance; fluorescence; J-aggregate.

\begin{abstract}
Polyaniline (PANI), is one of the promising candidates for utilization as electrode material in supercapacitors and its electro- and physical-chemical properties have been studied for several decades. However, there are fundamental questions that need to be answered. For example, is it possible to obtain and characterize individual PANI chains or what is the impact of
\end{abstract}


chains interactions on electro- and physical-chemical behavior? To that end, we isolated and characterized individual PANI chains alongside nanofibrillar assemblies for the first time, and moreover we demonstrate that the interaction between polyaniline chains and their assembly into nanofibrils have a crucial impact on electro- and physical-chemical behavior of PANI. Additionally, the optical transitions of three different conglomerations and protonation states are studied and correlated with the structural and electrochemical characterization. The mechanism of redox process for individual PANI chains and PANI nanofibrils is proposed. The specific capacitance of $1050 \mathrm{~F} / \mathrm{g}$ (at $25 \mathrm{~A} / \mathrm{g}$ of specific current) for 3-electrode configuration and $450 \mathrm{~F} / \mathrm{g}$ (at $9 \mathrm{~A} / \mathrm{g}$ of specific current) for 2-electrode configuration was obtained, which is the highest value reported to date.

\section{INTRODUCTION}

The storage of energy in semiconducting polymers or organic metals has been intensively investigated in the last few decades. ${ }^{1}$ It has been proposed that pseudocapacitance in these materials is associated with a sequence of redox processes that are highly reversible. ${ }^{2}$ Indeed, Conway and co-workers observed such behavior for the first time in $\mathrm{RuO}_{2} .{ }^{1}$ Among all semiconducting polymers, polyaniline (PANI) is probably one of the most important due to its simple synthesis, environmental stability and low synthetic cost. ${ }^{3}$ It was shown that depending on the method of polymerization (chemical or electrochemical) and choice of electrolytes and oxidants, PANI can be prepared as highly oriented fibrils,,${ }^{4,5}$ randomly assembled nanofibrils, ${ }^{6,7}$ or granules. The PANI structure is related to the assembly of polymer chains during the synthesis, which can be promoted by the formation of hydrogen bonds between water molecules and the nitrogen atoms. ${ }^{8,9}$ It has been shown that cyclic voltammetry of PANI exhibits generally 
two, ${ }^{9}$ but sometimes three, oxidation and reduction peaks. ${ }^{10}$ When the cyclic voltammograms exhibit two redox peaks, the first peak is related to emeraldine formation, at $360 \mathrm{mV}$ vs NHE, and the other, in the range of 650 to $800 \mathrm{mV}$ vs NHE, with the formation of pernigraniline. ${ }^{11} \mathrm{In}$ various polymer systems, the cyclic voltammograms exhibit three redox peaks, and the origin of the third (middle peak) is still under investigation; nevertheless, some authors attribute it to the cross-linking between the polymer chains. ${ }^{12}$ The number and positions of the redox peaks versus a reference electrode and the separation between peaks are important parameters in understanding the electrochemical processes taking place in PANI.

In this article, we report the electrochemical performance and analysis of electronic transitions of individual PANI chains and PANI nanofibrils. PANI was obtained by the method described in Experimental section. Briefly, aniline oxidation was interrupted after one hour by dilution followed by removal of reacted material through filtration and subsequent washing. Then PANI was immersed in solution of $5 \mathrm{M}$ formic acid. PANI nanofibrils (green) are precipitating on the bottom and individual PANI chains (and/or small associates) are released/stayed in the upper part of the solution (pink). This procedure allowed us to obtain individual PANI chains and polymer chains that can assemble into nanofibrils with diameters of $\sim 20 \mathrm{~nm}$. The polymer chains are oriented perpendicular to the direction of nanofibril propagation, as was proved in the literature. ${ }^{4,5}$ In this article, we obtained and studied for the first time the individual PANI chains and have recorded the unique redox process: a mechanism of the redox process is proposed below. Moreover, the individual PANI chains and suspension of PANI nanofibrils we synthesized fluoresce in an acidic medium ( pH 1.5), which contradicts the state-of-the-art research; the explanation for this unexpected behavior is given in detail later. Besides, the 
strongest fluorescence is measured for the individual PANI chains compare to the PANI nanofibrils in acidic or alkali medium, signaling an aggregation induced quenching mechanism.

\section{EXPERIMENTAL SECTION}

Analytic grade aniline, ammonium peroxydisulfate, formic acid, phosphoric acid and sodium chloride were used as received (Lachner, Czech Republic). Carbon cloth (Elat ${ }^{\circledR}$, Fuel Cells Etc. Co, USA) was used without further treatment.

2a. Synthesis of polyaniline: $0.004 \mathrm{~mol} \mathrm{~L}^{-1}$ aniline was dissolved in the solution of $5 \mathrm{~mol} \mathrm{~L}^{-1}$ formic acid and $6.4 \mathrm{~mol} \mathrm{~L}-1 \mathrm{NaCl}$ and then mixed with the solution containing $0.004 \mathrm{~mol} \mathrm{~L}^{-1}$ ammonium peroxydisulfate. The polymerization was performed for $60 \mathrm{~min}$ at $0^{\circ} \mathrm{C}$, after that it was diluted by $100 \mathrm{ml}$ of $5 \mathrm{~mol} \mathrm{~L}^{-1}$ formic acid and left for another 24 hours. The final product was filtered and washed by the solution of $5 \mathrm{~mol} \mathrm{~L}^{-1}$ formic acid. At the end of washing the suspension of PANI was obtained (3 wt. \%). PANI nanofibers precipitated and the upper solution contained isolated PANI chains. PANI nanofibers were used for the deposition on a $4 \times 3 \mathrm{~cm}$ carbon cloth sheet. The deposition of PANI nanofibrils on carbon cloth was performed by following method: 3 wt. $\%$ of PANI nanofibrils solution was placed on top of carbon cloth, the solvent was evaporated in the oven at $70^{\circ} \mathrm{C}$ to the constant weight. The amount of deposited PANI nanofibrils was $0.512 \mathrm{mg} / \mathrm{cm}^{2}$.

Cyclic voltammetry, galvanostatic charge/discharge were performed using an AUTOLAB PGSTAT302N potentiostat with FRA32M Module and Nova software 2.1. All measurements were performed at ambient temperature in $6 \mathrm{M} \mathrm{H}_{3} \mathrm{PO}_{4}$. The electrochemical impedance spectra (EIS) have been measured in the range from $10 \mathrm{kHz}$ to $\mathrm{m} 100 \mathrm{~Hz} \mathrm{mHz}$ with a $10 \mathrm{mV}$ amplitude, 
at potentials of $0,0.1,0.2,0.3,0.4,0.50 .6$ and $0.7 \mathrm{~V}$ vs $\mathrm{Ag} / \mathrm{AgCl}$. The Kronig-Kramers test was applied to verify the obtained EIS data.

The symmetrical capacitor (two-electrode setup) was fabricated using two identical electrodes with a foot-print area of $1 \mathrm{~cm}^{2}$ and a paper separator impregnated with the electrolyte solution. All measurements were performed within the potential range from 0 to $700 \mathrm{mV}$ vs $\mathrm{Ag} / \mathrm{AgCl}$.

\section{2b. Calculations}

Specific capacitances (C) for 3-electrode configuration was calculated from the data obtained by galvanostatic charge/discharge using the following equations:

$$
\mathrm{C}=\mathrm{I} \times \tau / \Delta \mathrm{V}
$$

where I - discharge current $(\mathrm{A} / \mathrm{g}), \tau$ - discharge time $(\mathrm{s}), \Delta \mathrm{V}$ - potential window excluding voltage drop (V).

Specific capacitances (C) for 2-electrode configuration (for one electrode) was calculated from the data obtained by galvanostatic charge/discharge using the following equations:

$$
\mathrm{C}=2 \times \mathrm{I} \times \tau / \Delta \mathrm{V}
$$

where I - discharge current $(\mathrm{A} / \mathrm{g}), \tau$ - discharge time $(\mathrm{s}), \Delta \mathrm{V}$ - potential window excluding voltage drop (V).

\section{2c. Characterization}

The scanning electron micrographs were obtained using a JEOL 6400 microscope. The transmission electron micrographs were obtained using a Tecnai G2 Spirit (FEI). 
The excitation dependent photoluminescence map was recorded on a Jasco FP-6200 model in quartz cuvettes.

FTIR spectra were recorded on a Perkin-Elmer PARAGON 1000 PC FT-IR spectrometer with resolution $4 \mathrm{~cm}^{-1}$ and accumulations: 16 and 32, in transmission mode, in $\mathrm{KBr}$ pellets.

UV-Vis spectra for all solutions were recorded on a Perkin-Elmer Lambda 20 UV-VIS spectrophotometer.

Matrix-assisted laser-desorption ionization time-of-flight mass spectra (MALDI-TOF MS) were obtained from a Bruker microflex MALDI-TOF spectrometer using Trans-2-[3-(4-tertButylphenyl)-2-methyl-2-propenylidene] malononitrile (DCTB) as a matrix in negative ionization mode.

X-ray photoelectron spectroscopy (XPS) was recorded on AXIS Supra photoelectron spectrometer (Kratos Analytical, UK).

\section{RESULTS AND DISCUSSION}

\section{3a. Materials characterization}

The formation of highly oriented PANI film from polyaniline suspension has previously been reported. ${ }^{4,5}$ The diameter of these fibrils was $30 \mathrm{~nm}$. In our work we modified the procedure proposed by Gospodinova N. et al. such that the polymerization reaction was interrupted by dilution followed by filtration and immersion/redistribution of obtained product again in formic acid to obtain isolated (individual) polymer chains and nanofibrils in one vessel, for more details see Scheme 1 and Figure 1d. In our experimental procedure we separate between two different 
structures, the green PANI nanofibrils that will precipitate after a short period of time and the individual PANI chains that will remain soluble in the upper part of the solution, coloring it pink.

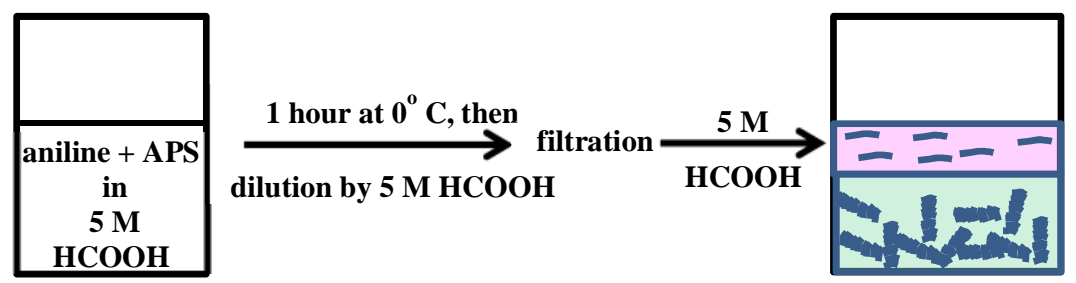

Scheme 1. Preparation of individual PANI chains and assembled PANI nanofibrils.

Formation of PANI nanofibrils was confirmed by transmission electron microscopy (TEM) shown in Figure 1f. The diameter of the nanofibrils was $\sim 20 \mathrm{~nm}$. Detailed TEM images are presented in the SI (Figure S1).

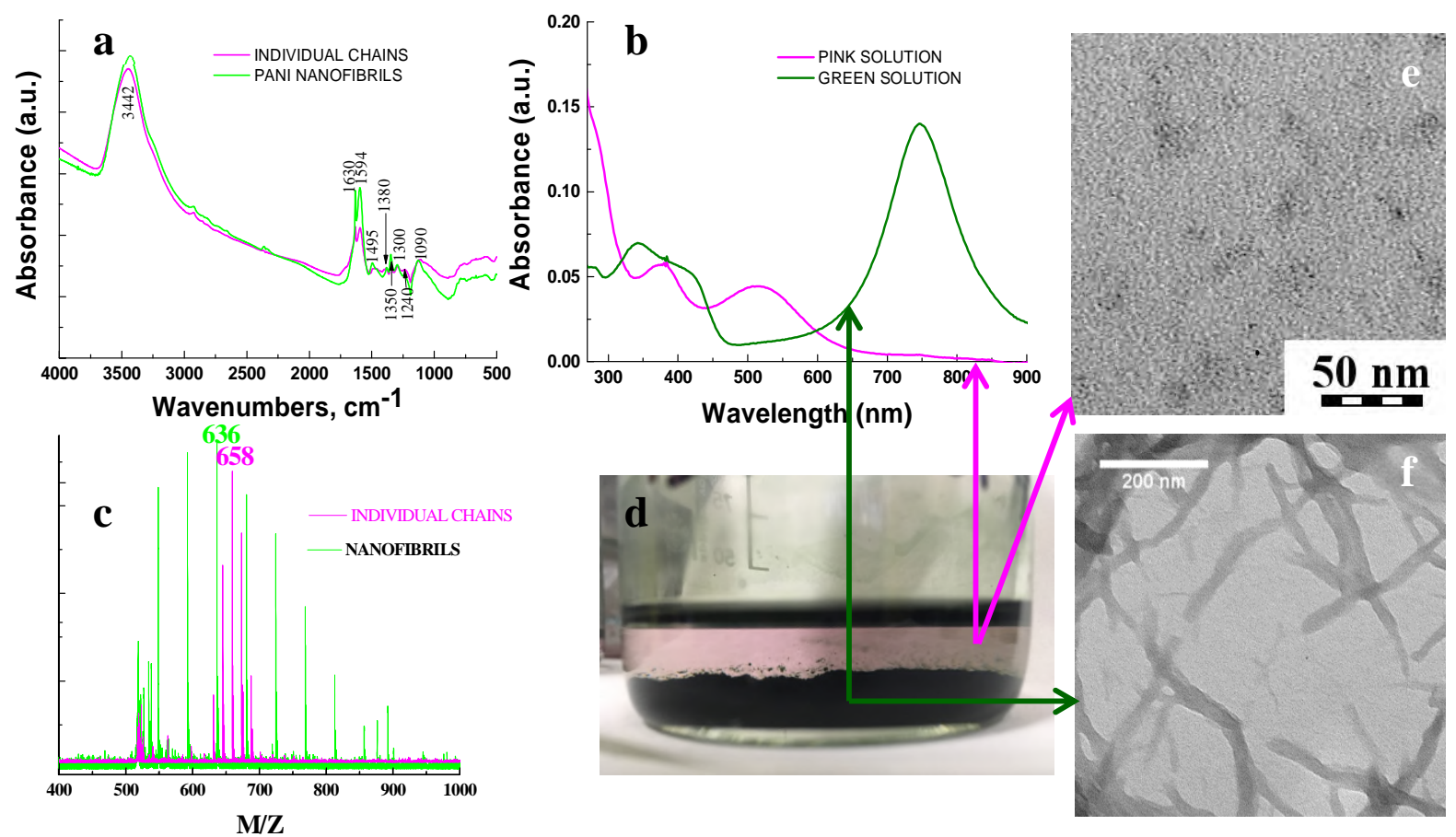


Figure 1. (a) FTIR spectra of individual PANI chains and PANI chains assembled into nanofibrils and (b) UV-Vis spectra of individual PANI chains and nanofibrils of PANI, and (c) MALDI ToF in negative ionization mode of individual PANI chains and nanofibrils of PANI. (d) pink solution of individual PANI chains and green solution of PANI nanofibrils in vessel. TEM images (e) of individual PANI chains and (f) PANI nanofibrils.

PANI nanofibrils were subsequently deposited on the surface of a carbon cloth (CC), which was further used as a current collector. It is clear from scanning electron microscopy (SEM) that the PANI nanofibrils did not have a specific orientation; they were randomly distributed on the surface of CC (Figure S3, SI).

\section{The suspension of isolated chains was studied with Transmission Electron Microscopy. A} representative TEM image is presented in Figure 1e (larger TEM image of the individual PANI chains is presented in the SI, Figure S1). It is revealed that there are no nanofibrils present; and irregular grans with a diameter of approximately $10 \mathrm{~nm}$ are observed. To determine the number of monomer units in PANI chains, MALDI ToF analysis, with trans-2-[3-(4-tert-Butylphenyl)-2methyl-2-propenylidene] malononitrile in ethyl acetate as matrix and the voltage used in the negative mode, is performed, see Figure 1c. For the individual PANI chains the mass-to-charge ratio has narrow distribution and value is $\mathrm{m} / \mathrm{z}=658$, and for PANI nanofibrils are $\mathrm{m} / \mathrm{z}=636$, which correspond to seven monomer units (theoretical value is 638). The higher value for individual PANI chains is probably connected with the attachment of water molecule to polymer chain (strong hydrogen interaction). The presence of water molecules is also confirmed by IR spectra (Figure 1a).The IR spectra of the individual PANI chains and assembled PANI nanofibrils are 
identical as can be seen on Figure 1a. However, the UV-Vis spectra for isolated PANI chains and assembled PANI nanofibrils are different (Figure 1b). The individual PANI chains possess only two peaks located at $370 \mathrm{~nm}$ and $520 \mathrm{~nm}$; on the other hand the assembled PANI nanofibrils have three peaks located at $340 \mathrm{~nm}, 410 \mathrm{~nm}$ and narrow sharp peak at $750 \mathrm{~nm}$ (long-wave absorption). The red-shift for assembled PANI nanofibrils (compared to the individual polyaniline chains) is associated with the formation of J-aggregates, leading to fibrillar selfassembly at the solid state. ${ }^{9,13}$ It has to be emphasized that UV-Vis spectra of individual polyaniline chains corresponds to the UV-Vis spectra of a dry, highly crystalline, PANI film, reported by us previously. ${ }^{9,14} \mathrm{We}$ can conclude (based on measured results) that the individual PANI chains and PANI nanofibrils have the same chemical structure but they are different in their organization: one is isolated polymer chains and the other is assembled nanofibrils. We present a schematic of the assembly process in Scheme 2.
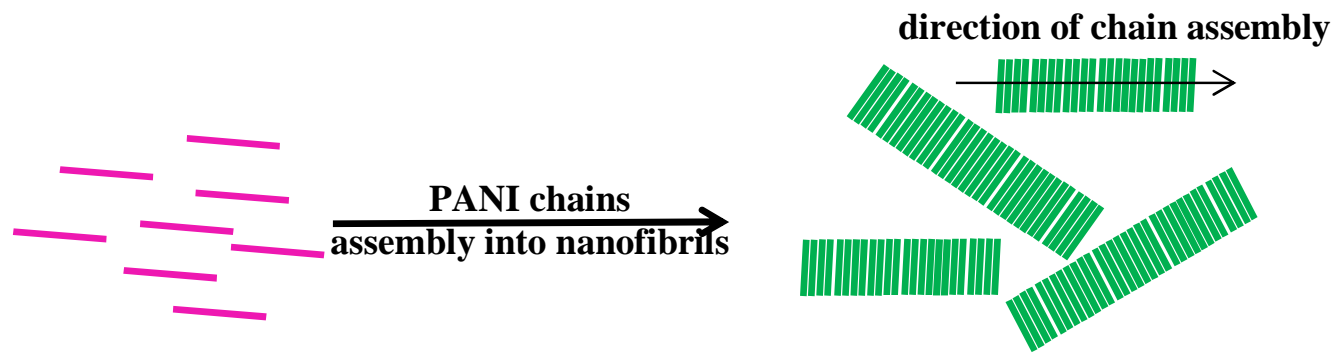

Scheme 2. A schematic representation of the assembly of individual PANI chains into PANI nanofibrils.

According to our knowledge, that this is the first time when the individual PANI chains are obtained through separation from a fibrillar assembly and extensively characterized. Yet, we have to mention the work of Andreatta and co-workers who dissolved PANI powder in the concentrated sulfuric acid with the formation of viscous solution of a purple-black colour. ${ }^{15}$ 
From our point of view, they obtained mixture of individual PANI chains and dispersed granules (as evidenced by UV-Vis spectra, presented in the article). ${ }^{15}$

Additionally, to prove that individual PANI chains and PANI nanofibrils consist from the same polymer chains, surface X-ray Photoelectron Spectroscopy (XPS) was performed. The results from XPS measurements are summarized and presented in Figure 2. It is demonstrated that both the individual PANI chains and PANI nanofibrils showed N 1s lines with similar binding energy at their peaks. After subtracting background, using Shirley method, spectra were fitted with Gaussian-Lorentzian functions and XPS Peak4.1 software. For an examination of obtained spectra, all the binding energy values were referenced to the $\mathrm{C} 1 \mathrm{~s}$ peak at $282.4 \mathrm{eV}$.

Deconvolution lead into four component peaks (Figure 2). The main peak of the fitted XPS curve is situated at $399.5 \mathrm{eV}$ for individual PANI chains and PANI nanofibrils, corresponding to uncharged nitrogen in PANI chains.
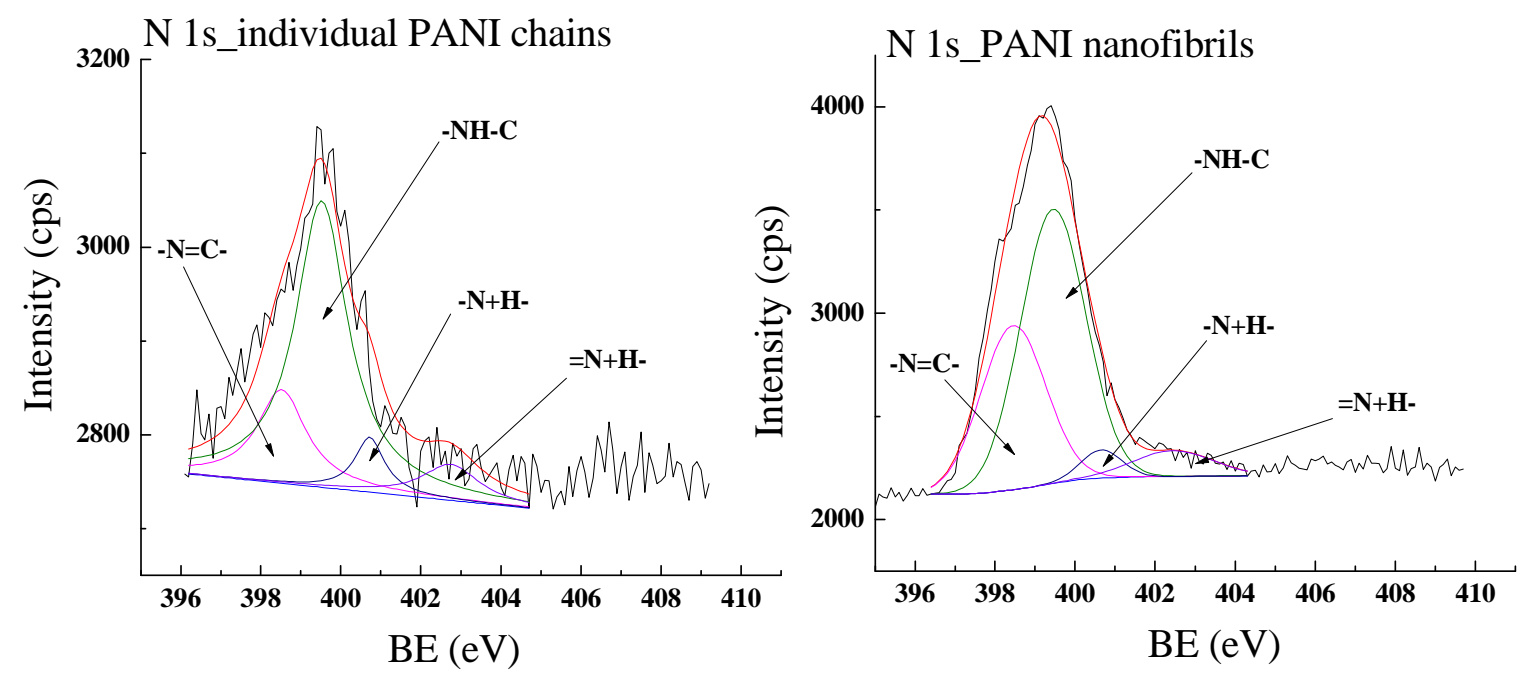

Figure 2. Core level spectra of $\mathrm{N} 1 \mathrm{~s}$ for individual PANI chains (left) and PANI nanofibrils (right). 
It is revealed from our analysis that the benzenoid amine is the main unit in polymer chain.

However, the ration of benzenoid amine units to quinoid imine units in individual PANI chains is higher compare to PANI nanofibrils, indicating that in individual PANI chains the benzenoid amine units are prevailing. Such fact has major impact on photoluminescence, which is presented and extensively discussed below.

\section{3b. Light emitting properties}

Water molecules hold a crucial role in assembling the PANI chains as was shown previously. ${ }^{4,5}$ Moreover, the presence of water has an essential impact on the photoluminescence of the PANI films, which was observed and shown for the first time by Alan G. MacDiarmid. ${ }^{3}$ It is generally understood that the fluorescent structure in the linear PANI is the benzenoid unit, and the quinoid units demonstrate no observable fluorescence. ${ }^{16}$ Moreover, it is a common phenomenon when in aqueous phase individual molecules fluorescent and become non-fluorescent in the solid state, and in general conductive polymeric materials are only weakly fluorescent without any distinctive features or long Stokes shifts. ${ }^{17}$ 

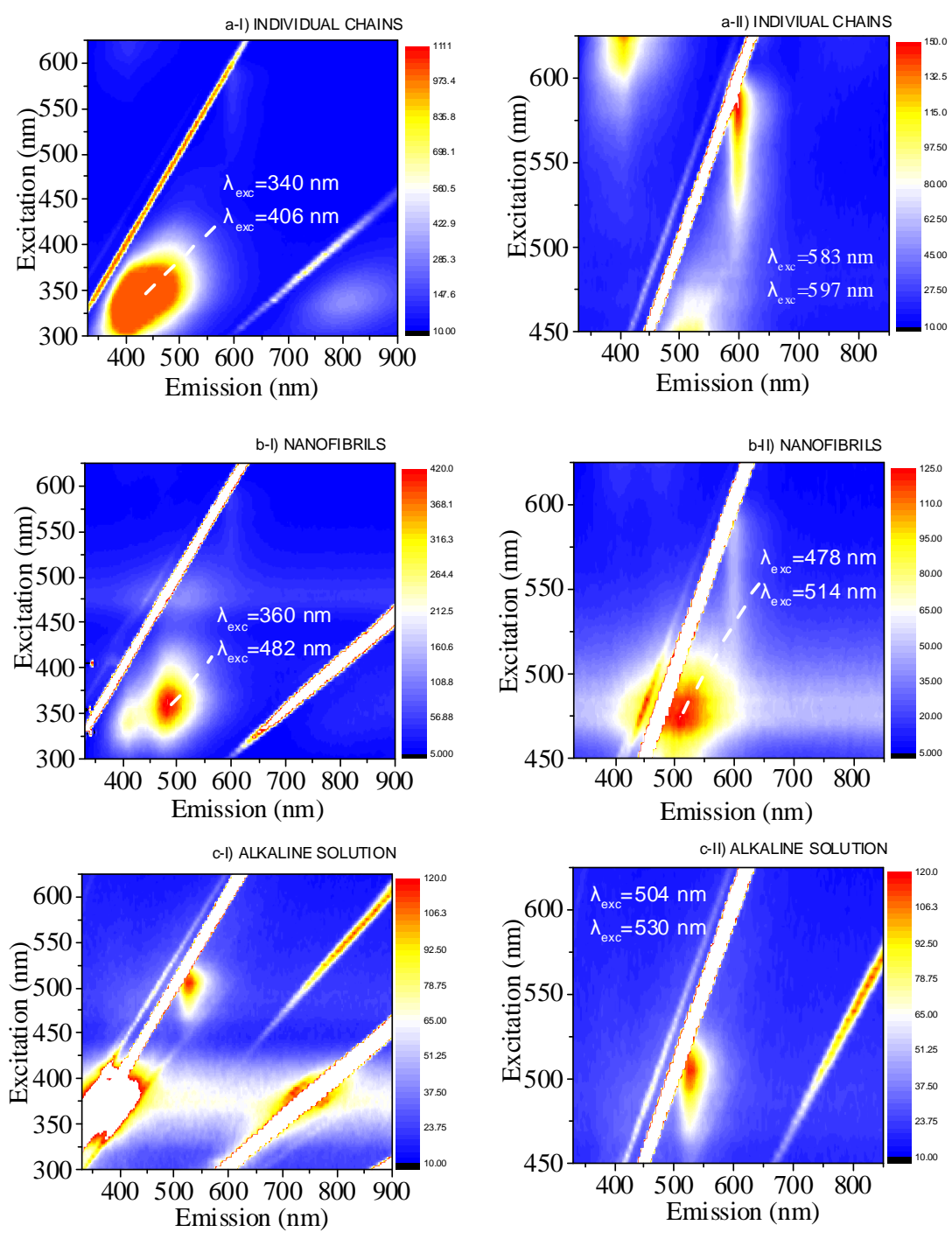

Figure 3. Photoluminescence spectrum of the (a) individual PANI chains (pink solution), (b) assembled PANI nanofibrils (green solution), (c) assembled PANI nanofibrils in alkali medium.

To further study the physical properties arising from the different arrangements and protonation states, we recorded photoluminescence excitation dependence maps for the three different samples, namely individual PANI chains, PANI nanofibrils, and PANI nanofibrils in basic pH. It 
structural and electrochemical analysis. The results are presented in Figure 3 and we summarize the excitation and emission wavelengths in Table 1. It is generally accepted that these materials are non-fluorescent, unless they will become cross-linked, for example through substitution with electron withdrawing groups such as perfluorinated chains. ${ }^{17}$ All Stokes shift (we observed) are modest, and the fluorescence is weak in the cases of the nanofibrils both in alkaline and acidic solution and appears to be stronger in the individual polyaniline chains. This result confirm the phenomenon when individual molecules (in our case individual polymer chains) fluorescence while a quenched fluorescence is observed in the aggregated state (formation of PANI nanofibrils). We summarize the optimum excitation and emission wavelengths in Table 1.

\begin{tabular}{|c|c|c|c|c|}
\hline Sample & $\begin{array}{c}\lambda_{\text {exc }} \\
\text { (blue) }\end{array}$ & $\begin{array}{c}\lambda_{\text {em }} \\
\text { (blue) }\end{array}$ & $\begin{array}{c}\lambda_{\text {exc }} \\
\text { (green) }\end{array}$ & $\begin{array}{c}\lambda_{\text {em }} \\
\text { (green) }\end{array}$ \\
\hline $\begin{array}{c}\text { Individual } \\
\text { chains }\end{array}$ & $340 \mathrm{~nm}$ & $406 \mathrm{~nm}$ & $583 \mathrm{~nm}$ & $597 \mathrm{~nm}$ \\
\hline Nanofibrils & $360 \mathrm{~nm}$ & $482 \mathrm{~nm}$ & $478 \mathrm{~nm}$ & $514 \mathrm{~nm}$ \\
\hline $\begin{array}{c}\text { Alkaline } \\
\text { solution }\end{array}$ & - & - & $504 \mathrm{~nm}$ & $530 \mathrm{~nm}$ \\
\hline
\end{tabular}

Table 1. In this Table we summarize the optimum excitation and emission wavelengths for the three tested samples.

It has to be emphasized that the photoluminescence spectra for individual PANI chains and PANI nanofibrils was measured at the $\mathrm{pH} \sim 1.5$. It was postulated that luminescence in polyaniline could be observed if the oxidized quinoid units and reduced benzenoid units formed a phase separated structure. ${ }^{3,19,20}$ Moreover, it was postulated that more than two benzenoid units 
not adjacent to a quinoid unit are needed for photoluminescence to be observed. ${ }^{3}$ Therefore, the individual PANI chains and assembled PANI nanofibrils we prepared must contain chains with more than two repeating benzenoid units not adjacent to a quinoid unit (which also was confirmed by XPS measurements, see Figure 2). Our analysis is showing that the as-synthesized PANI chains assembled into nanofibrils with water molecules incorporated between amino groups (due to hydrogen bonding), have extended benzenoid sequences arising from the linking between different polymer chains. The effect of water on the photoluminescence spectra of the PANI cast film was observed by Shimano et al. ${ }^{3}$ Thus, based on our UV-Visible measurements we concluded that our assembled PANI nanofibrils in its conducting form possesses photoluminescence due to the specific assembly of polymer chains, and with the help of water molecules, the requisite extended benzenoid sequence is formed. For further study of the conductive material see the electrochemical impedance measurements, Figure 6 . The photoluminescence spectrum is related to the HOMO/LUMO absorption transition, a change in the HOMO/LUMO energy gap, which is associated to interactions among the chains and between the chains and the solvent. The above factors would lead to a change in the photoluminescence spectrum. ${ }^{3,21}$ The energy gap for the $\pi$ - $\pi^{*}$ transition in the extended benzenoid sequence is decreased (excitation at $\lambda_{\mathrm{exc}}=478 \mathrm{~nm}$ ), and as a result, the emitted energy seen in the photoluminescence spectrum is also decreased $\left(\lambda_{\mathrm{em}}=514 \mathrm{~nm}\right)$. In contrast, Shimano et al. reported a peak in the photoluminescence spectrum of the conducting form at $467 \mathrm{~nm} \cdot{ }^{3} \mathrm{On}$ Figure 2c, we report the photoluminescence spectra of the PANI nanofibrils in alkali medium. A new emission peak $\lambda_{\mathrm{em}}=530 \mathrm{~nm}$ is recorded, which correspond to the optimum excitation wavelength $\lambda_{\text {exc }}=503 \mathrm{~nm}$ Figure $2 \mathrm{c}$. The emission peak is red-shifted compare to nanofibrils in acidic $\mathrm{pH}$, which can be explained by the formation of extended localized electron density. ${ }^{17}$ 


\section{3c. Electrochemical properties: cyclic voltammetry, galvanostatic charge/discharge and impedance spectroscopy.}

As was mentioned above, PANI fibrils have been reported, and their electrochemical performance has been studied; $6,7,22$ however, the shapes of their cyclic voltammograms described in the literature vary considerably, signaling a subtle influence from the macromolecular arrangements. We proposed that the shapes of cyclic voltammograms depend on the length of the PANI chains and more importantly on the interactions among the PANI chains. Abdelhamid et al. reported three peaks for PANI fibrils synthesized electrochemically. The average diameter of the fibrils was from $\sim 200 \mathrm{~nm}$ to $\sim 400 \mathrm{~nm} .{ }^{6}$ On the other hand, Zhang et al. showed only two adjacent peaks for PANI nanotubes obtained chemically with outer diameters from 170 to 260 nm. An explanation for this behavior was not given. ${ }^{7}$ Recently, Shi et al. reported the formation of coral-like PANI and its cyclic voltammogram: it is clear from the cyclic voltammograms that two redox peaks are located very close to each other. The authors explained the shift of the second peak in the negative direction by the presence of Sunset Yellow, which is used as a dopant and surfactant. ${ }^{22}$ From our point of view, such electrochemical behavior is related to the fact that PANI chains are assembled in a way where charge propagation is not kinetically and/or diffusional controlled. ${ }^{1}$ The PANI nanofibrils synthesized by our group (Figure 1f) possess only one major redox peak situated at $250 \mathrm{mV}$ for anodic and $90 \mathrm{mV}$ for cathodic curves $\mathrm{vs} \mathrm{Ag} / \mathrm{AgCl}$ reference electrode (at scan rate $30 \mathrm{mV} / \mathrm{s}$, in the potential window from 0 to $700 \mathrm{mV}$ ), which can be seen in the cyclic voltammograms presented in Figure $4 \mathrm{~b}$. The potential window 0 to $700 \mathrm{mV}$ has been chosen due to the stability of PANI and electrolyte. 
The cyclic voltammetry for PANI nanofibrils in the potential window from $-200 \mathrm{mV}$ to $800 \mathrm{mV}$ is presented in the SI (Figure S4). In the case of the nanofibrils, the cyclic voltammetry demonstrated only one redox peak in a similar pattern with the individual chains.
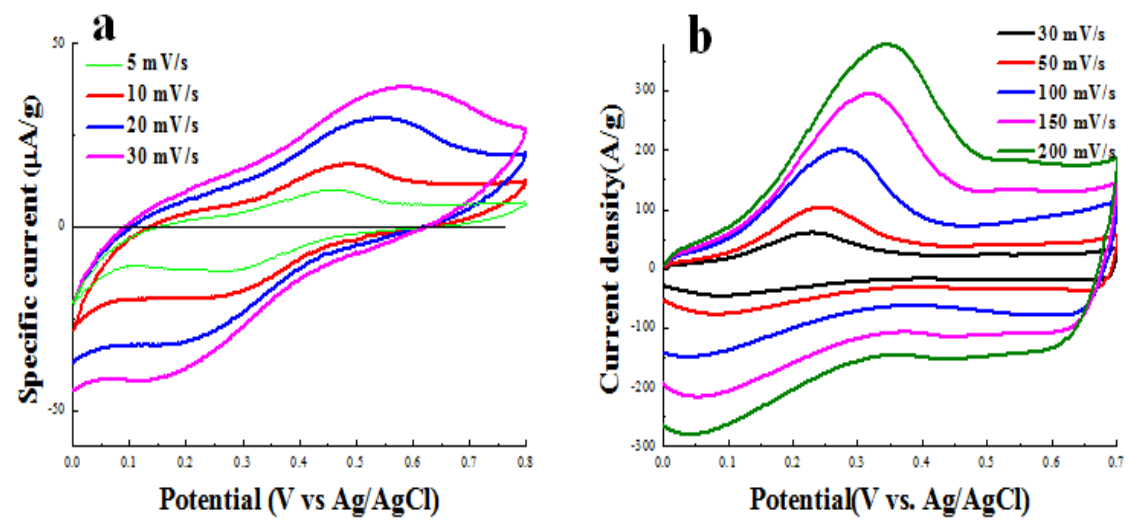

Figure 4. Cyclic voltammetry scans of individual PANI chains deposited on FTO (a) and (b) PANI nanofibrils deposited on CC.

The electrochemical behavior of individual PANI chains deposited on FTO electrode is presented in Figure 4a. It is demonstrated that individual PANI chains possess only one redox peak located at $470 \mathrm{mV}$ for anodic and $260 \mathrm{mV}$ for cathodic curves respectively (scan rate 5 $\mathrm{mV} / \mathrm{s}$; potential window from 0 to $800 \mathrm{mV}$ ). The value for anodic peak (470 mV vs $\mathrm{Ag} / \mathrm{AgCl})$ corresponds to the open circuit potential. We can conclude that individual PANI chains and assembled PANI nanofibrils possess only one redox peak, which contradicts the state-of-the-art research (most of the published data present two or more redox peaks). The difference in the position of the redox peaks between individual PANI chains and PANI nanofibrils is explained by the fact that assembled PANI nanofibrils have extended electronic interactions between 
polymer chains promoted by water molecules, also proved by UV-Vis spectra (appearing longwave absorption, Figure 1b). That is why the oxidation and reduction peaks for PANI nanofibrils are shifted to negative direction (meaning that electrochemical process is facile). On the basis of the previous consideration, we proposed that unique electrochemical behavior (only one redox peak) is connected both with nature of redox process for individual PANI chains and with the assembly of the PANI chains and the interactions of the polymer chains with each other; these effects are crucial for the electrochemical performance. The separation between anodic and cathodic peaks is $210 \mathrm{mV}$ for the individual PANI chains and $160 \mathrm{mV}$ for the assembled PANI nanofibrils. From common point of view, the position of the redox peaks, their separation, and the number of peaks gives us direct information about the interactions between PANI chains. We halted the synthesis after one hour, and by doing this, we could obtain PANI nanofibrils with diameters of $\sim 20 \mathrm{~nm}$ with a very narrow size distribution (see Figure 1d and Figure S1, for details). The presence of only one redox peak for PANI nanofibrils have been reported in the literature, but the authors did not give any explanation for the observed phenomenon. ${ }^{23}$ Also, when the PANI fibrils are large (dimeter $\sim 200 \mathrm{~nm}$ or higher), the cyclic voltammogram exhibits three peaks. ${ }^{6}$ We propose that the second and the third peaks are the results for redox processes of PANI phases with different chains assemblies; if the process is facile, the new peak will be located next to the first one, but when the redox process is controlled by diffusion or kinetics, the peak will shift to more positive potentials. Therefore, the large PANI fibrils contain domains of both chains assemblies - one with facile charge propagation between polymer chains and the other with limited charge propagation.

The impact of the assembly of PANI chains into nanofibrils on the electrochemical performance is demonstrated by the dependence of the specific capacitance versus specific current (Figure $5 \mathrm{c}$ 
and 5f) for PANI nanofibrils deposited on CC, measured in 3-electrode and 2-electrode configurations.
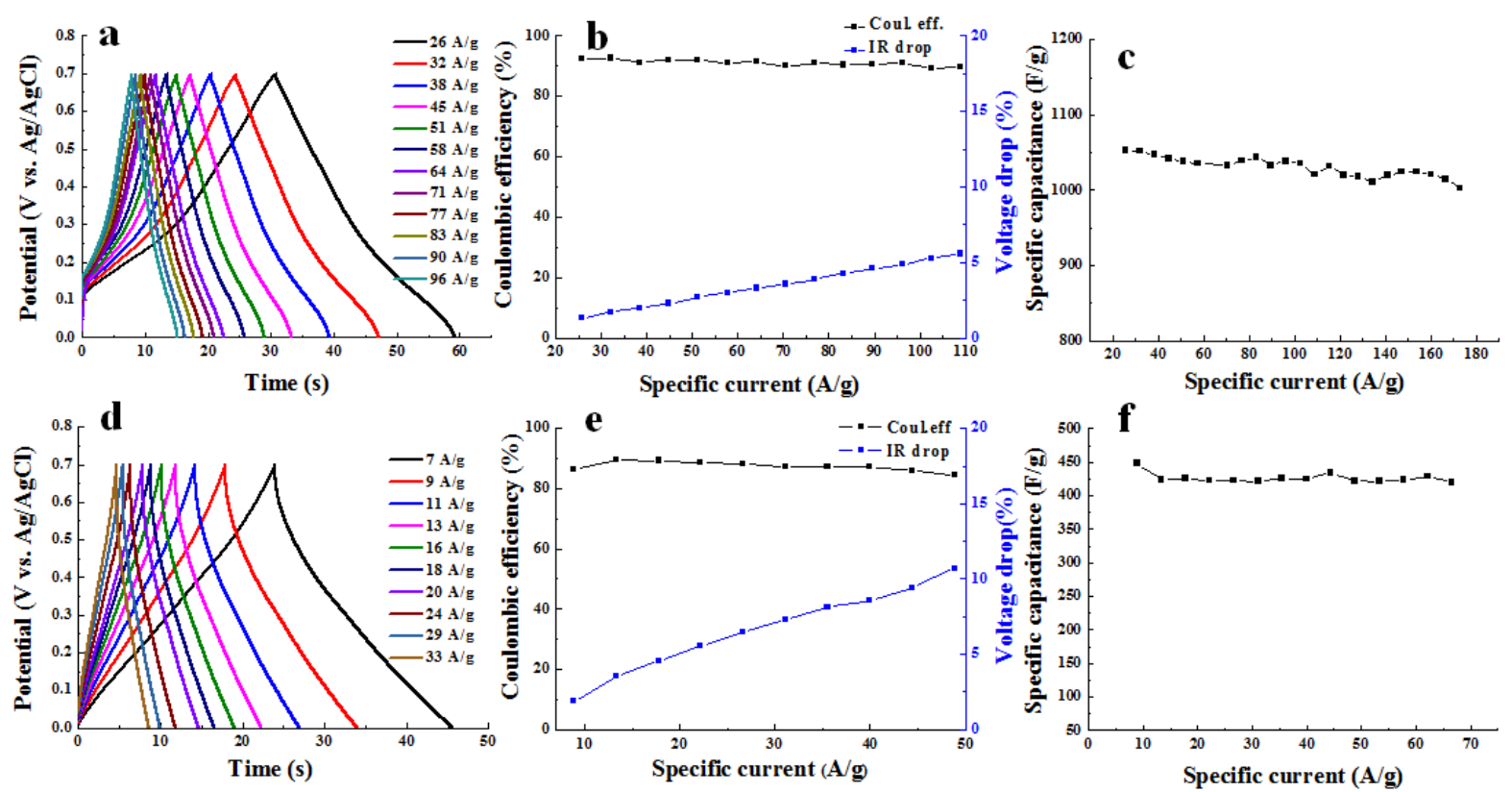

Figure 5. Galvanostatic charge-discharge, Coulombic efficiency, IR drop and specific capacitance versus specific current for 3-electrode configuration (a), (b) and (c); and 2-electrode configuration (d), (e) and (f) respectively.

\section{Specific capacitance and coulombic efficiency were calculated from galvanostatic}

\section{charge/discharge measurements presented in Figure 5a and 5d.}

In the case of pseudocapacitive device, the redox processes have to be facile (charge propagation is not kinetically and/or diffusional controlled), and preferably the whole electroactive material should participate in energy storage and delivery. ${ }^{1,24-25}$ Galvanostatic charge/discharge 
measurements were taken on a 3-electrode configuration and on symmetrical pseudocapacitor (2electrode configuration), and all electrochemical characteristics (such as specific capacitance, coulombic efficiency, IR drop) were calculated from the obtained results. It is obvious from Figure $5 \mathrm{c}$ and $4 \mathrm{f}$ that when the specific current increased by almost a factor of seven, the specific capacitance only decreased by $10 \%$, from $1050 \mathrm{~F} / \mathrm{g}$ to $1000 \mathrm{~F} / \mathrm{g}$ for 3-electrode configuration and from $453 \mathrm{~F} / \mathrm{g}$ to $410 \mathrm{~F} / \mathrm{g}$ for 2-electrode configuration.

Such behavior could be explained by the nature of the electroactive material; PANI chains are assembled into nanofibrils in which charge propagation is not-limiting factor, which is why with increasing charge/discharge current the specific capacitance only decreased by $10 \%$. Such statement is also confirmed by the dependence of Coulombic efficiency and IR drops versus specific current, presented in Figure $5 \mathrm{~b}$ and $5 \mathrm{e}$ for various electrode configurations.

The electrochemical impedance spectroscopy has been conducted for different applied constant potentials - from PANI in its reduced state $(0 \mathrm{~V})$ to PANI in its oxidized state $(0.7 \mathrm{~V})$, see Figure 6. These data clearly demonstrate that at each applied potential, complex plan plots represent only the resistance of the electrolyte but not the polymer in its real part. The resistivity of the polymer, even in its reduced state, is lower than that of the electrolyte. Such behavior can again be explained by the formation of extended benzenoid sequences generated from PANI chains (formation of PANI nanofibrils) and promoted by water molecules. Notably, it has been reported that "The conductivity of the phase of reduced repeat units is higher than that of the phase of oxidized repeat units. ${ }^{19}$ This finding supports our hypothesis that reduced units (extended benzenoid sequence) not only have low resistivity (confirmed by impedance) but also possess photoluminescence and have charge propagation that is not kinetically and/or diffusional controlled (confirmed by cyclic voltammetry). 


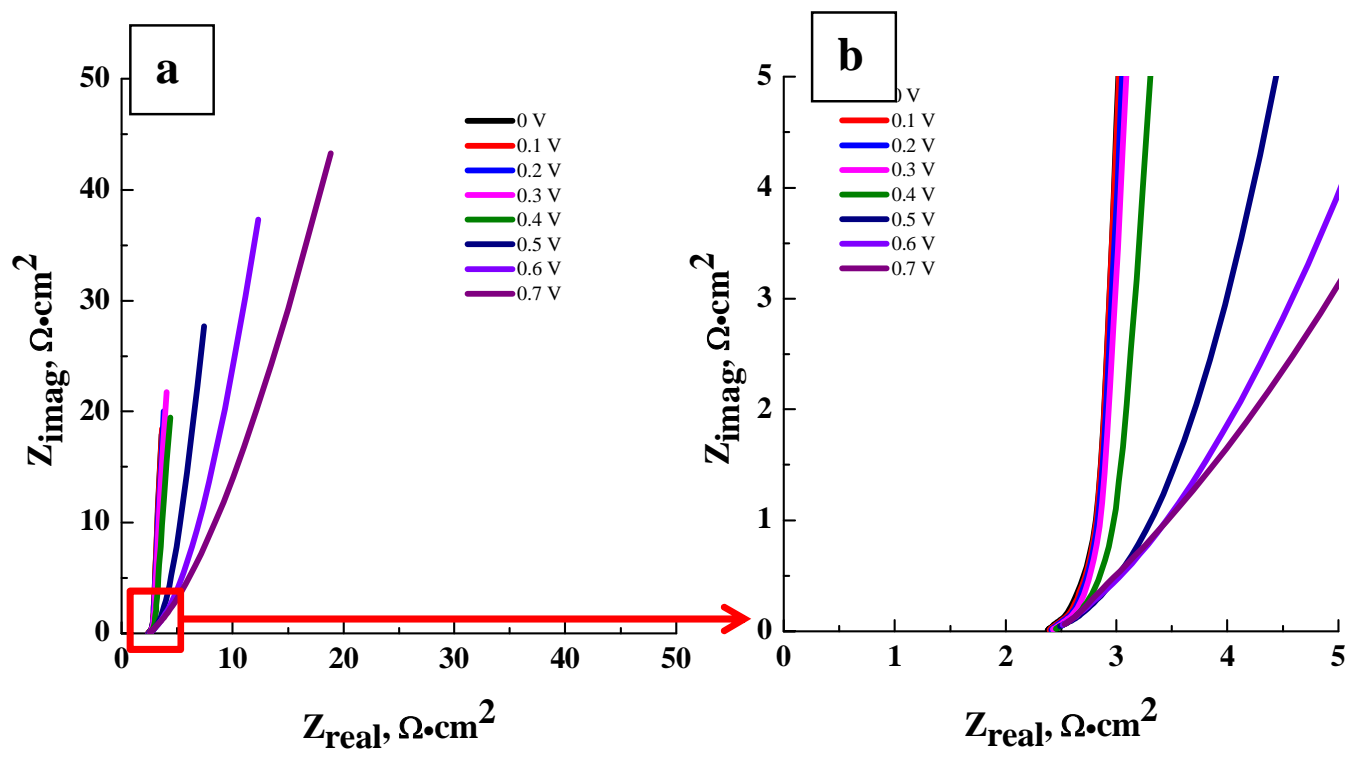

Figure 6. Electrochemical impedance spectroscopy curves (a) and the corresponding magnification (b), of PANI nanofibrils deposited on CC, measured at various constant applied potential.

\section{CONCLUSIONS}

In summary, we demonstrate for the first time that the interaction between isolated PANI chains and their assembly into nanofibrils have a crucial impact on electro- and physical-chemical behavior of the material. Electrochemical studies showed that PANI nanofibrils exhibit only one redox peak, in a similar manner with the individual PANI chains. When the charge propagation is limited by kinetics or diffusion, the appearing of second peak that is shifts to more positive potentials (also the peak separation increases) is observed, and in some cases, a third peak appears. From our point of view, the appearance of such peaks is only related to the formation of PANI phases with various chains assemblies. Phases with facile charge propagation demonstrate only one major peak, while on the other hand phases with limited charge propagation have two 
and more peaks. When both phases are present, three or more peaks may be detected in the cyclic voltammograms. PANI nanofibrils, reported in this article, possess not only one redox peak but also the symmetrical pseudocapacitor obtained from this active material possesses a specific capacitance of $\sim 450 \mathrm{~F} / \mathrm{g}$ in the specific current range from 10 to $70 \mathrm{~A} / \mathrm{g}$, which is the highest value reported up to now.

\section{ASSOCIATED CONTENT}

Supporting Information. Detailed TEM images of the PANI nanofibrils and individual PANI chains, SEM of PANI nanofibrils deposited on CC, and cyclic voltammetry in the potential range from $-200 \mathrm{mV}$ to $800 \mathrm{mV}$ vs $\mathrm{Ag} / \mathrm{AgCl}$ are available free of charge via the internet.

\section{AUTHOR INFORMATION}

\section{Corresponding Author}

*Elena Tomšík, e-mail: tomsik@imc.cas.cz.

ORCID:

Tomšík E: 0000-0002-1749-1091

Bieloshapka I.: 0000-0002-8727-2505

Ivanko I.: 0000-0003-1898-8662

Dallas P.: 0000-0002-1920-3399

\section{Author Contributions}


The manuscript was written through contributions of all authors. All authors have given approval to the final version of the manuscript.

\section{Notes}

The authors declare no competing financial interest.

\section{ACKNOWLEDGMENT}

We acknowledge Czech Republic Grand Agency (grant number: 15-14791S) and OPPK (CZ.2.16/3.1.00/21545) from the European Regional Development Fund. We also would like to acknowledge Hromádková J. for the SEM and TEM measurements.

\section{REFERENCES}

(1) B. E. Conway Electrochemical Supercapacitors: Scientific Fundamentals and Technological Applications, Kluwer Academic, New York, USA 1999.

(2) Conway B. E. Transition from "Supercapacitor" to "Battery" Behavior in Electrochemical Energy Storage. J. Electrochem. Soc., 1991, 138, 6, 1539-1548.

(3) Shimano J. Y.; MacDiarmid A.G. Polyaniline, a Dynamic Block Copolymer: Key to Attaining its Intrinsic Conductivity? Synth. Met., 2001, 123, 251-262.

(4) Gospodinova N.; Ivanov D. A.; Anokhin D. V.; Mihai I.; Vidal L.; Brun S.; Romanova J.; Tadjer A. Unprecedented Route to Ordered Polyaniline: Direct Synthesis of Highly Crystalline Fibrillar Films with Strong $\pi-\pi$ Stacking Alignment. Macromol. Rapid Commun., 2009, 30, 29-33.

(5) Gospodinova N.; Dorey S.; Anokhin D.; Ivanov D.; Romanova J.; Kolev H. (Centre National de la Recherche Scientifique and Universite de Haute-Alsace). Method of 
preparing polyaniline films and highly self-oriented films obtained Patent US 20110281098 A1, 2011.

(6) Abdelhamid M. E.; Snook G. A.; O’Mullane A. P. Electrochemical Tailoring of Fibrous Polyaniline and Electroless Decoration with Gold and Platinum Nanoparticles. Langmuir, 2016, 32, 8834-8842.

(7) Zhang L.; Peng H.; Zujovic Z. D.; Kilmartin P. A.; Traves-Sejdic J. Characterization of Polyaniline Nanotubes Formed in the Presence of Amino Acids. Macromol. Chem. Phys., 2007, 208, 1210-1217.

(8) Gospodinova N.; Tomsik E. Hydrogen-Bonding versus $\pi-\pi$ Stacking in the Design of Organic Semiconductors: From Dyes to Oligomers. Prog. Polym. Sci., 2015, 43, 33-47.

(9) Gospodinova N.; Tomšík E.; Omelchenko O. J-Like Liquid-Crystalline and Crystalline States of Polyaniline Revealed by Thin, Highly Crystalline, and Strongly Oriented Films. J. Phys. Chem. B, 2014, 118, 8901-8904.

(10) Focke W. W.; Wnek G. E.; Wei Y. Influence of Oxidation State, pH, and Counterion on the Conductivity of Polyaniline. J. Physic. Chem., 1987, 91, 22, 58135818.

(11) Macdiarmid A. G.; Chiang J.-C.; Halpern M.; Huang W.-S.; Mu S.-L.; Somasiri N. L. D.; Wu W.; Yaninger S. I. Electrochemical Characteristics of "Polyaniline" Cathodes and Anodes in Aqueous Electrolytes. Mol. Cryst. Liq. Cryst., 1985, 121, 173.

(12) Genies E. M.; Lapkowski M.; Penneau J. F. Cyclic Voltammetry of Polyaniline: Interpretation of the Middle Peak. J. Electroanal. Chem., 1988, 249, 97-107.

(13) Tempelaar R.; Jansen T. L. C.; Knoester J. Exciton-Exciton Annihilation Is Coherently Suppressed in H-Aggregates, but Not in J-Aggregates. J. Phys. Chem. Lett., 2017, 8, 6113-6117. 
Omelchenko O.; Tomsik E.; Zhigunov A.; Guskova O.; Gribkova O.;

Gospodinova N. J-Like Supramolecular Assemblies of Polyaniline in Water. Macromol. Chem. Phys., 2014, 118, 29, 8901-8904.

Andreatta A.; Cao Y.; Chiang J. C.; Heeger A. J.; Smith P. Electrically-

Conductive Fibers of Polyaniline Spun from Solutions in Concentrated Sulfuric Acid.

Synth. Met., 1988, 26, 383-389.

(16) Kim K.; Lin L. B.; Ginder J. M.; Gustafson T. L. Luminescence and Picosecond Photoinduced Absorption of Polyaniline. Epstein A. J. Synth. Met., 1992, 49-50, 423428.

(17) Dommett M.; Rivera M.; Crespo-Otera R. How Inter- and Intramolecular Processes Dictate Aggregation-Induced Emission in Crystals Undergoing Excited-State Proton Transfer. J. Phys. Chem. Lett., 2017, 8, 6148-6153.

(18) Dallas P.; Rašović I.; Porfyrakis K. Mapping and Tuning the Fluorescence of Perfluorinated Polyanilines Synthesized through Liquid-Liquid Interfaces. J. Phys. Chem. B., 2016, 120 (13), 3441-3454.

(19) Chen S.-A.; Chuang K.-R.; Chao C.-I.; Lee H.-T. White-Light Emission from Electroluminescence Diode with Polyaniline as the Emitting Layer. Synth. Met., 1996, $82,207-210$.

(20) Lee H.-T.; Chuang K.-R.; Chen S.-A. Conductivity Relaxation of 1-Methyl-2pyrrolidone-Plasticized Polyaniline Film. Macromolecules, 1995, 28, 7645-7652.

D. Phillips, Polymer Photophysics: Luminescence, Energy Migration and Molecular Motion in Synthetic Polymers, Chapman \& Hall, Cambridge, 1985.

Shi M.; Zhang Y.; Bai M.; Li B. Facile Fabrication of Polyaniline with Coral-Like Nanostructure as Electrode Material for Supercapacitors. Synth. Met., 2017, 233, 74-78. 
(23) Liu T.; Finn L.; Yu M.; Wang H.; Zhai T.; Lu X., Tong Y.; Li Y. Polyaniline and Polypyrrole Pseudocapacitor Electrodes with Excellent Cycling Stability. Nano Letters, 2014, 14, 2522-2527.

$$
\text { Comway B. E.; Pell W.G. Power Limitations of Supercapacitor Operation }
$$

Associated with Resistance and Capacitance Distribution in Porous Electrode Devices. $J$. Power Sources, 2002, 105, 169-181.

(25) Gao S.; Zhang L.; Qiao Y.; Dong P.; Shi J.; Cao S. Electrodeposition of Polyaniline on Three-Dimensional Graphene Hydrogel as a Binder-Free Supercapacitor Electrode with High Power and Energy Densities. RCS Ad., 2016, 6, 58854-58861.

\section{TOC GRAPHICS}

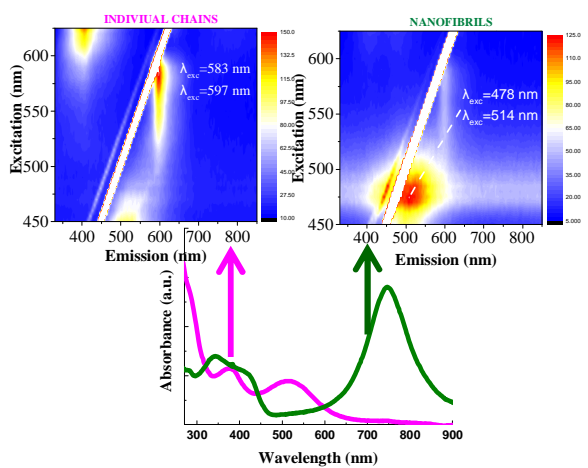

